\title{
Interactions of UHE cosmic ray nuclei with radiation during acceleration: consequences for the spectrum and composition
}

\author{
D. Allard ${ }^{1}$ and R. J. Protheroe ${ }^{2}$
}

\author{
${ }^{1}$ Laboratoire Astroparticule et Cosmologie (APC), Université Paris 7/CNRS, 10 rue A. Domon et L. Duquet, \\ 75205 Paris Cedex 13, France \\ e-mail: denis.allard@apc.univ-paris7.fr \\ 2 Department of Physics, The University of Adelaide, SA 5005, Australia \\ e-mail: rprother@physics.adelaide.edu.au
}

Received 12 February 2009 / Accepted 28 May 2009

ABSTRACT

\begin{abstract}
In this paper, we study the diffusive shock acceleration of cosmic-ray protons and nuclei, taking all the relevant interaction processes with photon backgrounds into account. We investigate how the competition between protons and nuclei is modified by such acceleration parameters as the acceleration rate, its rigidity dependence, the photon density, and the confinement capability of the sources. We find that protons are likely to be accelerated to higher energies than nuclei in the case of interaction-limited acceleration processes, whereas nuclei are accelerated to higher energies than protons for confinement-limited acceleration. Finally, we discuss our results in the context of possible astrophysical accelerators and in the light of recent cosmic-ray data.
\end{abstract}

Key words. acceleration of particles - nuclear reactions - nucleosynthesis, abundances

\section{Introduction}

By plotting magnetic field vs. size of various astrophysical objects, Hillas (1984) identified possible sites of acceleration of UHE CR based on whether or not the putative source could contain the gyro-radius of the accelerated particles, and on the likely velocity of scattering centers in these sites, and narrowed the field of possible sources to radio galaxy lobes and galaxy clusters. This issue has been re-examined recently in the context of diffusive shock acceleration (DSA) (Protheroe 2004) taking account of energy losses (synchrotron) and interactions (Bethe-Heitler and pion photoproduction) which can cut the spectrum off, and so provide additional constraints. It was found that with the maximum possible acceleration rate for a given magnetic field the spectrum is cut off by pion photoproduction if the magnetic field is below $\sim 3 \mu \mathrm{G}$, and for higher fields the spectrum is cut off by synchrotron losses.

However, for an acceleration rate $10^{-4}$ times the maximum possible, pion photoproduction cuts off the spectrum for fields below $\sim 100 \mu \mathrm{G}$ and synchrotron losses cut off the spectrum for higher fields. In order to accelerate protons above $10^{20} \mathrm{eV}$ the ideal sources were found to have acceleration region sizes (magnetic field) between $1 \mathrm{kpc}(\sim 1 \mathrm{mG})$ and $1 \mathrm{Mpc}(\sim 1 \mu \mathrm{G})$ such that the gyro-radius can be contained within the accelerator. These parameters allow the spectrum to extent beyond $10^{20} \mathrm{eV}$ where it will be cut off either by synchrotron losses (for the higher magnetic fields in this range) or by pion photoproduction (for the lower fields in this range). This makes radio galaxies and clusters of galaxies the probable sites of UHE CR acceleration (see Fig. 6 of Protheroe 2004) in agreement with the conclusion of Hillas. However, if the whole acceleration region is moving relativistically, Doppler boosting in energy of neutrons (produced by pion photoproduction) escaping from such sources allows blazar jets (Protheroe et al. 2003) and GRB (Waxman 2006 and references therein) to remain possible sites of UHE CR acceleration, and in these cases proton synchrotron losses are likely to cut off the spectrum. Large-scale cosmic shocks in the Universe may also be sites of UHE CR acceleration (Kang \& Jones 2002; Inoue et al. 2007) as well as colossal magneto-hydrodynamic fossil AGN structures (Benford \& Protheroe 2008), AGN jets and cocoons surrounding AGN jets (Schopper et al. 2002; Norman et al. 1995) and hot spots in radio lobes of powerful radio galaxies (Rachen \& Biermann 1993). In these cases pion photoproduction, the size of the accelerator or the time available for acceleration may cause the cut-off in the spectrum of protons at acceleration. In a recent review, Biermann et al. (2008) concluded that the most promising contenders are radio galaxies and GRB.

Apart from the nature of the cosmic accelerators, the composition of the accelerated particles at the highest energies is a key parameter in understanding the origin of the extragalactic cosmic-rays and the physical conditions at play at the sources. To determine the spectrum and composition of accelerated particles at an astrophysical source the injected composition at the shock and the interactions within the acceleration region should be considered. Satellite measurements of low energy galactic cosmic-rays show that the cosmic-ray composition is deficient in $\mathrm{H}$ with respect to interstellar matter, and the relative abundances of the various species are believed to be related to their volatility (Meyer et al. 1997). It is of course possible that such enrichment takes place also in extragalactic sources.

In the present paper, we take into account all the relevant interactions of protons and nuclei with ambient photon backgrounds and calculate the spectrum and composition resulting from diffusive shock acceleration for various compositions injected at the shock. Using a box model of DSA (Protheroe 2004), the SOPHIA event generator for pion photoproduction (Mücke et al. 2000) and the photon-nucleus interaction event generator 


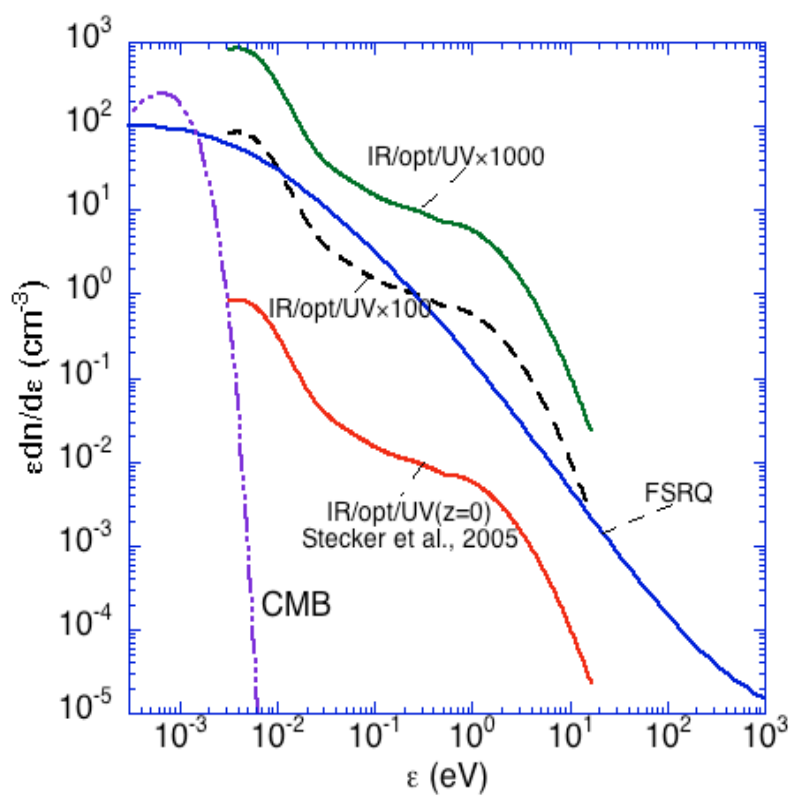

Fig. 1. Radiation field of the CMB compared with the IR/Opt/UV from Stecker et al. (2005) with various scaling factors. Also shown, as will be discussed in Sect. 4, is the radiation field expected at $200 \mathrm{kpc}$ along the jet from the flat-spectrum radio quasar (FSRQ) 0208-512 based on its spectral energy distribution given in Fig. 5 of Tavecchio et al. (2002).

developed in Allard et al. (2005-2008), we study the dependence of the composition and spectrum of accelerated cosmic rays on the acceleration rate, the rigidity dependence of the diffusion coefficient, the confinement within the acceleration site, and the density of the photon backgrounds. The paper is organized as follows, in the next section we introduce the relevant interaction processes of protons and nuclei with photon backgrounds. In Sect. 3 we describe the box model for DSA we use in our calculations. In Sect. 4 we present the spectra and composition resulting from the acceleration processes under different hypotheses about the injected composition and physical parameters for the accelerators. Finally, in Sect. 5 we discuss our results.

\section{Interactions of nucleons and nuclei with radiation and their modeling}

Protons and nuclei propagating through the intergalactic medium interact mainly with cosmic microwave background (CMB), infrared, optical and ultraviolet (IR/Opt/UV) photon backgrounds. These interactions produce features in the propagated UHE CR spectrum such as the "GZK cutoff" (Greisen 1966; Zatsepin \& Kuzmin 1966). Pions produced in the same interactions generate the cosmogenic neutrino flux (Berezinsky \& Zatsepin 1969). During the acceleration process, these interactions are also expected to take place at a rate depending on the ambient photon density and can also produce spectral and composition features in the cosmic-rays as well as secondary particles (neutrinos and photons), as discussed by Protheroe (2004) in detail for the case of proton primaries.

In the following, we will use the recent estimate of Stecker et al. (2005) for the intergalactic background to model the photon backgrounds in the infra-red, optical and ultra-violet window. We re-scale its density with different scaling factors to account for the higher density IR/opt/UV backgrounds that could be expected in astrophysical sources (see Fig. 1).
Bethe-Heitler pair production interactions with the various photon backgrounds are treated as a continuous energy loss process. We use the cross sections, inelasticities and the mass and charge scaling of the attenuation length for nuclei provided by Rachen (1996). If the energy of the background photons exceeds $\sim 145 \mathrm{MeV}$ in the nucleon rest frame, protons and neutrons can interact through the pion photoproduction process. This high inelasticity process is better treated by Monte Carlo calculation, and our Monte Carlo code uses the SOPHIA event generator (Mücke et al. 2000) allowing us to treat accurately the various interaction channels (direct pion production, resonances, multipion production) and their branching ratios (see Rachen 1996). The energy dependence of the mean free path of protons for photopion production with $\mathrm{CMB}$ and IR/opt/UV photons is shown in Fig. 2a. Also shown is the mean free path for a IR/opt/UV background 100 times denser.

The interactions experienced by nuclei with photon backgrounds differ from those of protons. In addition to the pair production losses that result in a decrease of the Lorentz factor and the rigidity of the nucleus, one must consider the photo-disintegration (also called photo-erosion) processes that leads to the ejection of one or several nucleons from the nucleus. Different photo-erosion processes dominate the total interaction cross section at different energies (Puget et al. 1976). The lowest energy disintegration process is the Giant Dipole Resonance (GDR) which results in the emission of one or two nucleons and $\alpha$ particles. The GDR process is the most relevant as it has the highest cross section and the lowest thresholds, between 10 and $20 \mathrm{MeV}$, for all nuclei. For nuclei with mass $A \geq 9$, we use the theoretical GDR cross sections presented by Khan et al. (2005), which take into account all the individual reaction channels $(n, p, 2 n, 2 p, n p, \alpha, \ldots)$ and are in better agreement with data than previous treatments. For nuclei with $A<9$, we use the phenomenological fits to the data provided by Rachen (1996). Between $\sim 30 \mathrm{MeV}$ in the nucleus rest frame and the photopion production threshold the quasi-deuteron (QD) process becomes comparable to the GDR, and dominates the total cross section at higher energies.

Pion photoproduction (baryonic resonance (BR) process) by nuclei becomes relevant above $150 \mathrm{MeV}$ in the nucleus rest frame, i.e., $\sim 5 \times 10^{21} \mathrm{eV}$ in the lab frame for iron nuclei interacting with the CMB. We use the parameterization given by Rachen (1996) in which the cross section in this energy range is proportional to the mass of the nucleus. The basis for this scaling that of the deuteron photoabsorption cross section which is known in great detail, although nuclear shadowing effects are expected to break this scaling above $1 \mathrm{GeV}$. It is important to note that pion photoproduction cross sections for nuclei are different from the free nucleon case. In particular, in nuclei the baryonic resonances heavier than the first $\Delta$ resonance are far less pronounced than for nucleons, and the cross sections can not be simply derived from the free nucleon case. We also follow Rachen (1996) for the treatment of secondary particle production, nucleon multiplicities, energy losses, and the probability of absorption of the produced pion by the parent nucleus.

The Lorentz-factor dependence of the total mean free path (all photo-disintegration processes and all channels) for photodisintegration for four different abundant species is shown in Fig. $2 \mathrm{~b}$ for the same photon backgrounds as Fig. 2a. One can see that the interaction thresholds occur at similar Lorentz factors for all the species, and that the mean free paths scale approximately linearly with the mass as a consequence of the mass scaling of the cross sections. Photo-disintegration is dominated by the GDR process over the whole energy range, except for the 

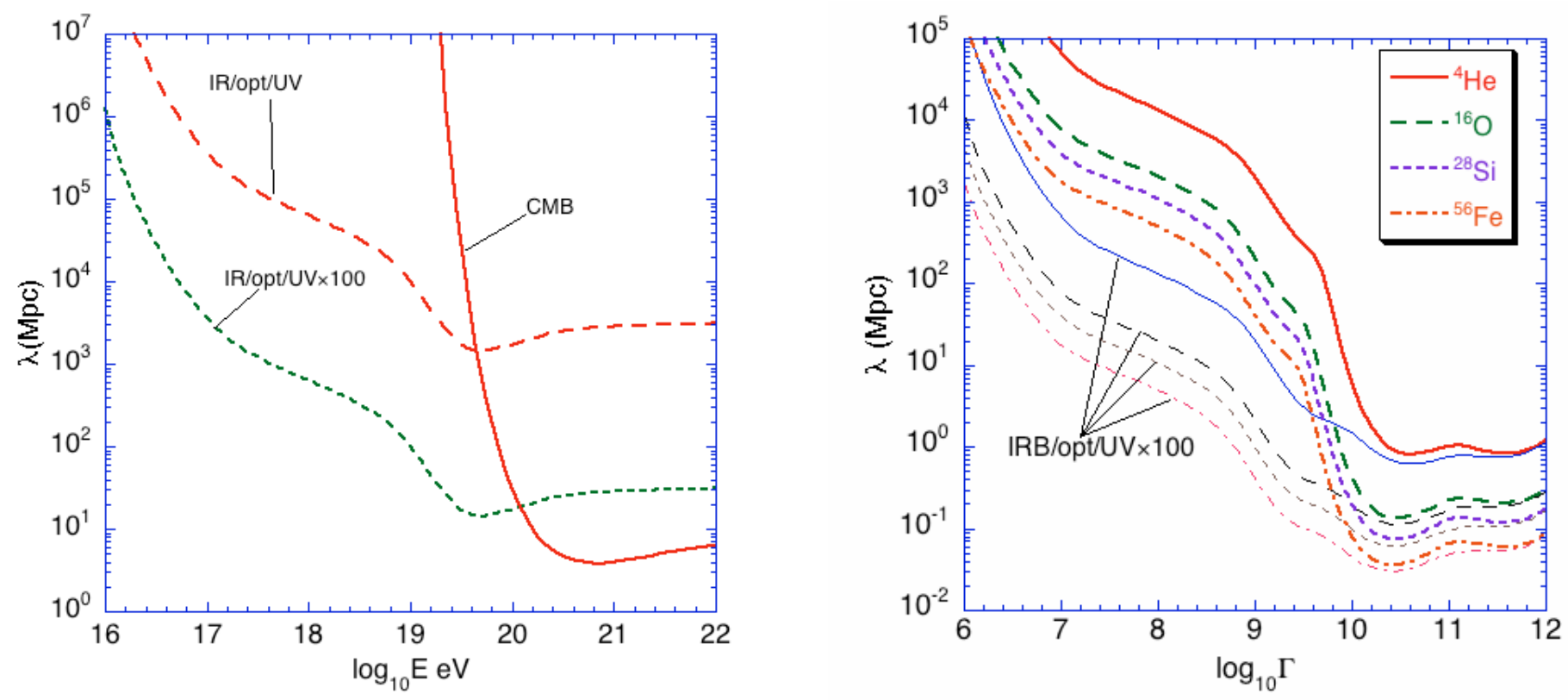

Fig. 2. a) Left: energy dependence of the mean free path for pion photoproduction of cosmic-ray protons in the intergalactic background radiation fields, and for the case where the IR/Opt/UV background is 100 times higher. b) Right: Lorentz-factor dependence of the mean free path for photo-disintegration for four different nuclei $(\mathrm{He}, \mathrm{O}, \mathrm{Si}, \mathrm{Fe})$ in the same photon backgrounds as in the left panel.

second minimum at the highest energies which is due to the BR process (see Fig. 3b of Allard et al. 2006 for the contributions of the various processes to the mean free path).

\section{Diffusive shock acceleration}

Diffusion of energetic particles in magnetic fields depends on their magnetic rigidity $\rho \equiv p c / Z$ e, where $p$ is the the particle's momentum and Ze its charge. In DSA the rigidity gain and escape rates depend on the diffusion coefficient which is usually assumed to have a power-law dependence on rigidity, $\kappa(\rho) \propto \rho^{\delta}$, with the exponent depending on the nature of the turbulence present in the magnetic field: $\delta=1 / 3$ (Kolmogorov spectrum), $1 / 2$ (Kraichnan spectrum) or 1 (completely disordered field). The diffusion coefficient is lowest, and the acceleration fastest, for the case where the magnetic field $B$ is highly disordered, and it can approach the Bohm diffusion coefficient, $\kappa_{\mathrm{B}}=\frac{1}{3} R_{g} c$ where $R_{\mathrm{g}}=\rho / c B$ is the gyro-radius. In this case Jokipii (1987) showed that if the diffusion coefficient parallel to the magnetic field direction is a factor $\eta>1$ times the Bohm limit, i.e.

$\kappa_{\|}=\eta \frac{1}{3} r_{g} c$

then the diffusion coefficient perpendicular to the magnetic field is expected to be approximately

$\kappa_{\perp} \approx \frac{k_{\|}}{1+\eta^{2}}$

provided that $\eta$ is not too large (values in the range up to 10 appear appropriate).

Under this assumption, and for the case of DSA at a strong plane parallel shock (i.e. with magnetic field $B$ parallel to the shock normal) with shock speed $u_{1}$, this leads to a rigidity gain rate

$\left.r_{\text {gain }}(\rho) \equiv \frac{1}{\rho} \frac{\mathrm{d} \rho}{\mathrm{d} t}\right|_{\text {gain }} \approx \frac{3 u_{1}^{2} B}{20 \eta} \rho^{-1}$, and for a perpendicular shock (i.e. with magnetic field perpendicular to the shock normal) the rigidity gain rate would be a factor $\sim 2.5 \eta\left(1+\eta^{2}\right)$ higher (Protheroe 1998).

In general, for particle acceleration by electric fields induced by the motion of magnetic fields $B$ (including those at astrophysical shocks), the maximum rigidity gain rate of relativistic particles can be written

$r_{\text {gain }}=\xi(\rho) c^{2} B \rho^{-1}$.

where $\xi(\rho)<1$ is the acceleration rate parameter which depends on the details of the acceleration mechanism, with $\xi=1$ corresponding to the highest posible gain rate for any mechanism. For DSA $\xi(\rho)$ will depend on the shock velocity, magnetic field alignment and diffusion coefficients. For example, for the parallel shock case with $u_{1}=0.1 c$ and $\eta=10$ one finds $\xi(\rho) \leq 1.5 \times 10^{-4}$, and for the equivalent perpendicular shock case $\xi(\rho) \leq 0.04$ (see Protheroe 1998). Detailed and rigorous treatments of DSA are given in several review articles (Drury 1983; Blandford \& Eichler 1987; Berezhko \& Krymsky 1988), see particularly the review by Jones \& Ellison (1991), on the plasma physics of shock acceleration, which also includes a brief historical review and refers to early work.

Protheroe \& Stanev (1999) proposed a box model of DSA (based on that developed by Szabo \& Protheroe 1994) which was able to reproduce the essential features of DSA in a simple leaky-box scenario. Particles were injected into the "box", and while inside the box their energy increased at the average rate that would apply given the physical parameters of the shock acceleration being modelled, i.e. the upstream and downstream diffusion coefficients, the shock velocity and compression ratio. Particles being accelerated were made to leak out of the box, again at the average rate that would apply given the physical parameters, and these particles represented the accelerated particles escaping downstream in the standard shock acceleration picture. Protheroe (2004) incorporated improvements suggested by Drury et al. (1999) into the model, and used it to investigate cut-offs and pile-ups in spectra of accelerated particles in the presence of energy losses or interactions, including the case of 


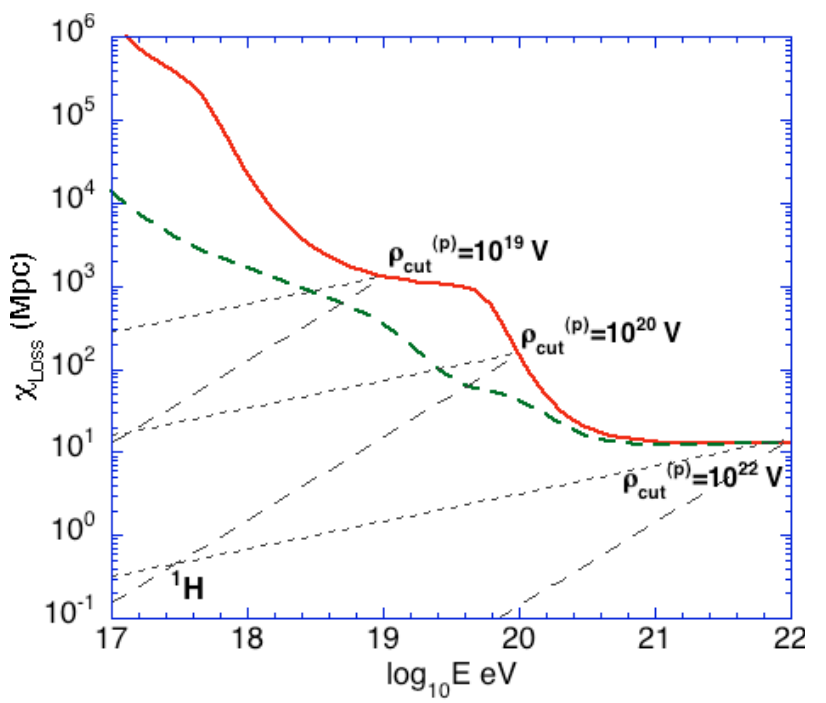

Fig. 3. Energy dependence of the loss length for cosmic-ray protons in the CMB plus intergalactic IR/Opt/UV background (continuous line) and assuming a IR/Opt/UV background 100 times higher (dashed line). The loss length is compared to the gain length assuming three different values for $\rho_{\text {cut }}^{(p)}\left(10^{19}, 10^{20}\right.$ and $\left.10^{22} \mathrm{~V}\right)$ and $\delta=1 / 3$ (thin short dashed lines) and $\delta=1$ (thin long dashed lines).

acceleration and propagation of UHE cosmic ray protons in the presence of the CMB. It was found that although almost all information about the acceleration conditions was lost after propagation, as a result of the GZK cut off, significant information remained imprinted in the spectrum of cosmogenic neutrinos produced in the "GZK interactions". In the present paper we use the calculation scheme of Protheroe (2004) as it is very convenient to implement in a numerical or Monte Carlo program to investigate the spectrum and composition of UHE cosmic rays accelerated in the presence of the $\mathrm{CMB}$ and IR/Opt/UV radiation fields.

As we have seen, the normalization of the gain rate depends in a non-trivial way on the magnetic field, turbulence spectrum, shock configuration and shock velocity. Different classes of source, and even different sources of the same class, will accelerate particles up to different maximum energies. We have therefore arbitrarily chosen to normalize the gain rate at a given cut-off rigidity to that necessary for protons to reach that given cut-off rigidity in a steady state situation in the presence of the $\mathrm{CMB}$ and IR/Opt/UV radiation. We have done this irrespective of the rigidity dependence of $r_{\text {gain }}(\rho)$ for two reasons: firstly because we are interested in the maximum energies achieveable and in interactions occurring at or near cut-off, and secondly because it is only the gain rate close to the cut-off rigidity, not the rigidity-dependence of the gain rate, that determines the value of the cut-off rigidity, at least for a steady-state situation.

The nominal cut-off rigidity for protons $\rho_{\text {cut }}^{(p)}$ occurs where the gain rate equals the loss rate in the intergalactic IR/Opt/UV background,

$r_{\text {gain }}\left(\rho_{\text {cut }}^{(p)}\right)=r_{\text {loss }}\left(\rho_{\text {cut }}^{(p)}, Z=1\right)$.

In Fig. 3 we show the energy dependence of the loss (or attenuation) length for cosmic-ray protons, $x_{\text {loss }}(\rho, Z=1)$, in the $\mathrm{CMB}$ plus intergalactic IR/Opt/UV background (continuous line) and in the CMB plus an intergalactic IR/Opt/UV background 100 times higher (dashed line) (assuming the same photon backgrounds as in Fig. 2). We have added to Fig. 3 curves

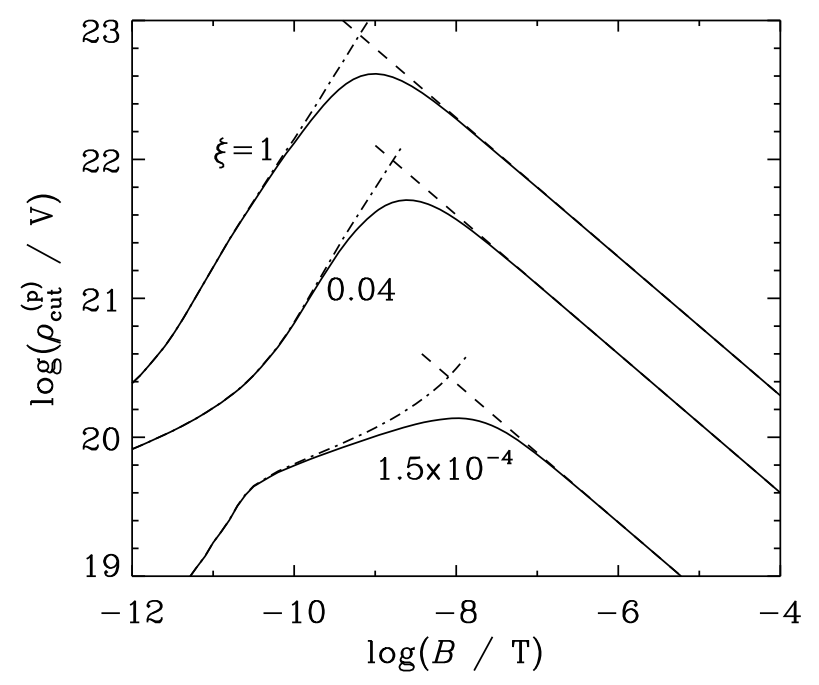

Fig. 4. Cut-off rigidity of protons accelerated in the presence of CMB radiation vs. magnetic field for $\xi=1,0.04$ and $1.5 \times 10^{-4}$ : chain curve cutoff due to Bethe-Heitler pair production and pion photoproduction; dashed curve - cutoff due to synchrotron; solid curve - total.

representing $x_{\text {gain }}(\rho) \equiv c / r_{\text {gain }}(\rho)$ for different values of $\rho_{\text {cut }}^{(p)}$ (assuming $\delta=1 / 3$ and $\delta=1)$. Using the value of $\rho_{\text {cut }}^{(p)}$ in the CMB plus intergalactic IR/Opt/UV background to define the normalization of the rigidity gain rate we have

$r_{\text {gain }}(\rho)=r_{\text {gain }}\left(\rho_{\text {cut }}^{(p)}\right)\left(\frac{\rho}{\rho_{\text {cut }}^{(p)}}\right)^{-\delta}=r_{\text {loss }}\left(\rho_{\text {cut }}^{(p)}, Z=1\right)\left(\frac{\rho}{\rho_{\text {cut }}^{(p)}}\right)^{-\delta}$

We have chosen for $\rho_{\text {cut }}^{(p)}$ values in the range $10^{19} \mathrm{~V}$ to $10^{22} \mathrm{~V}$, and we now discuss for what magnetic fields and acceleration rate parameters it is feasible for protons to achieve these cutoff rigidities in the $\mathrm{CMB}$ plus IR/Opt/UV radiation. For $\rho_{\text {cut }}^{(p)}=$ $10^{19}, 10^{20}, 10^{22}$ and $10^{22} \mathrm{~V}$, the corresponding values of $\xi\left(\rho_{\text {cut }}^{(p)}\right) B$ are $1.5 \times 10^{-15}, 6.9 \times 10^{-14}, 6.9 \times 10^{-12}$ and $1.6 \times 10^{-10} \mathrm{~T}$. However, for a given value of the product $\xi\left(\rho_{\text {cut }}^{(p)}\right) B$, not all vales of $B$ are possible as protons will lose energy also by synchrotron radiation, and this actually becomes more important than pion photoproduction losses at high rigidity. If the proton spectrum is cut-off by synchrotron loss, then the cut-off rigidity is given by

$\rho_{\text {cut }}^{(p)}=2 \times 10^{18} \xi^{1 / 2} B^{-1 / 2} \mathrm{~V}$

where $B$ is in tesla. To illustrate this, we plot in Fig. 4 the cut-off rigidity vs. magnetic field taking into account of synchrotron, Bethe-Heitler pair production and pion photoproduction losses for three values of acceleration rate parameter. For example, for the parallel shock case with $u_{1}=0.1 c$ and $\eta=10$ for which $\xi(\rho) \approx 1.5 \times 10^{-4}$ it is possible for protons to reach rigidities above $\rho=10^{19} \mathrm{~V}$ for magnetic fields in the range $5 \times 10^{-12}$ to $5 \times 10^{-6} \mathrm{~T}$, and to reach rigidities above $\rho=10^{20} \mathrm{~V}$ fields in the range $10^{-9}$ to $5 \times 10^{-8} \mathrm{~T}$ are required with the maximum rigidity possible for that case being $1.5 \times 10^{20} \mathrm{~V}$ achievable for $10^{-8} \mathrm{~T}$. For the equivalent perpendicular shock case for which $\xi(\rho) \approx 0.04, \rho=10^{21} \mathrm{~V}$ can be reached for magnetic fields in the range $10^{-10}$ to $2 \times 10^{-7} \mathrm{~T}$, with the maximum rigidity possible being $5 \times 10^{21} \mathrm{~V}$ achievable for $10^{-9} \mathrm{~T}$. Higher rigidities require relativistic shocks or different mechanisms, but proton rigidities above $4 \times 10^{22} \mathrm{~V}$ are are not possible without Doppler boosting of neutrons produced in pion photoproduction within a relativistically moving source such as an AGN jet or GRB. 

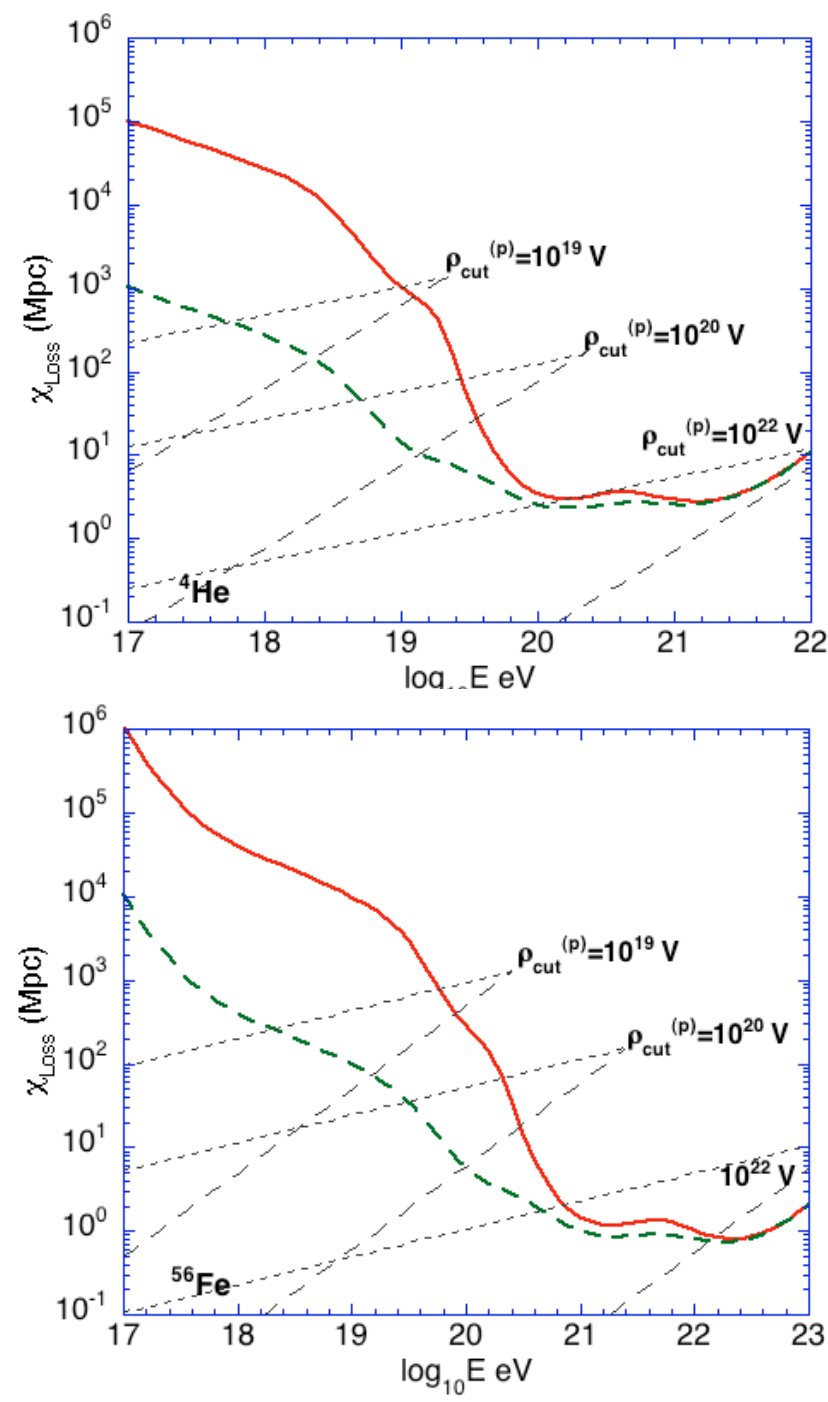

Fig. 5. As Fig. 3 but for a): He (top), and b): Fe nuclei (bottom).

In Fig. 5 the energy dependence of the loss length for He (Fig. 5a) and Fe (Fig. 5b) is plotted (assuming the same photon backgrounds as in Fig. 2) and is compared to the energy dependence of gain lengths for the same rigidity dependences as the curves in Fig. 3. As the abscissa refers to the energy, and the acceleration depends on rigidity, the curves for the gain length for a given $\rho_{\text {cut }}^{(p)}$ are shifted in energy proportionally to the charge (i.e. by a factor of 2 for $\mathrm{He}$ and a factor of 26 for $\mathrm{Fe}$ ). Although the loss length is not strictly relevant for nuclei because nuclei do not remain on the same energy loss curve when losing energy, unlike protons, these figures show that the output of the acceleration process will crucially depend on the normalization of the background radiation and the gain rate and its rigidity dependence, and that the "competition" between protons and nuclei will, at least for some combinations of parameters, be non-trivial.

\subsection{Box model approximation}

In the box model of shock acceleration, particles of magnetic rigidity $\rho_{0}$ are injected into the acceleration zone, or "box", and while inside the box are accelerated at a rate $r_{\text {tot }}(\rho)$ and escape from the box at a rate $r_{\text {esc }}(\rho)$. These two rates uniquely determine the spectrum of accelerated particles, i.e. those escaping from the box.

The average rate of change of rigidity of a nucleus of rigidity $\rho$ and atomic number $Z$ during shock acceleration in the presence of energy losing processes is

$$
\left.\frac{\mathrm{d} \rho}{\mathrm{d} t}\right|_{\text {total }, Z} \equiv \rho r_{\text {tot }}(\rho, Z)=\rho\left[r_{\text {gain }}(\rho)-r_{\text {loss }}(\rho, Z)\right]
$$

where $r_{\text {loss }}(\rho, Z)$ includes both losses due to interactions such as Bethe-Heitler pair production that can be approximated as continuous energy losses, and pion photoproduction in which a significant fraction of a proton's energy is lost per interaction. Hence, using Eq. (6) we have

$r_{\text {tot }}(\rho, Z)=r_{\text {loss }}\left(\rho_{\text {cut }}^{(p)}, Z=1\right)\left(\frac{\rho}{\rho_{\text {cut }}^{(p)}}\right)^{-\delta}-r_{\text {loss }}(\rho, Z)$.

If there are no energy losses and no additional escape processes a power-law spectrum results, and the integral spectral index , $(\Gamma-1)$, equals the ratio of the escape rate to the gain rate. In this case, the escape rate representing escape downstream is $(\Gamma-1) r_{\text {gain }}(\rho)$, and for injection of $N_{0}$ particles of rigidity $\rho_{0}$ the expected differential rigidity spectrum of accelerated particles is

$\Phi_{0}(\rho)=N_{0}(\Gamma-1) \rho_{0}^{\Gamma-1} \rho^{-\Gamma}$.

The acceleration zone extends distances $L_{1}(\rho)=\kappa_{1}(\rho) / u_{1}$ and $L_{2}(\rho)=\kappa_{2}(\rho) / u_{2}$ upstream and downstream from the shock, respectively where $u_{1}$ and $u_{2}$ are the upstream and downstream flow velocities in the shock frame. Adding an extra escape term $r_{\mathrm{esc}}^{\max }\left(\rho, \rho_{\max }\right)$ responsible for the "maximum rigidity" for example arising from the finite size of the accelerator, and an escape term $r_{\text {esc }}^{\text {fallout }}(\rho)$ resulting from energy losses causing particles to "fall out of the box" gives the total escape rate

$$
\begin{aligned}
r_{\mathrm{esc}}(\rho)=(\Gamma & -1) r_{\mathrm{loss}}\left(\rho_{\mathrm{cut}}^{(p)}, Z=1\right)\left(\frac{\rho}{\rho_{\mathrm{cut}}^{(p)}}\right)^{-\delta} \\
& +r_{\mathrm{esc}}^{\max }\left(\rho, \rho_{\max }\right)+r_{\mathrm{esc}}^{\text {fallout }}(\rho, Z) .
\end{aligned}
$$

For the case of diffusion (with diffusion coefficient $\kappa \propto \rho^{\delta}$ ) perpendicular to the shock normal to the edge of the acceleration region being responsible for the maximum rigidity,

$r_{\mathrm{esc}}^{\max }\left(\rho, \rho_{\max }\right)=(\Gamma-1) r_{\text {loss }}\left(\rho_{\text {cut }}^{(p)}, Z=1\right)\left(\frac{\rho_{\max }^{2}}{\rho \rho_{\text {cut }}^{(p)}}\right)^{-\delta}$.

For the escape rate resulting from energy losses

$r_{\text {esc }}^{\text {fallout }}(\rho, Z)=\left.\frac{1}{L(\rho)} \frac{\mathrm{d} L_{2}}{\mathrm{~d} \rho} \frac{\mathrm{d} \rho}{\mathrm{d} t}\right|_{\text {loss }, Z}=\delta \ell_{2} r_{\text {loss }}(\rho, Z)$,

where $L=\left(L_{1}+L_{2}\right)$, and we adopt $\ell_{2} \approx 0.5$ (Protheroe 2004).

Interactions of relativistic particles with matter or radiation generally result in the interacting particle losing energy, and this energy lost is either given to the struck particle or photon (elastic collision) or used in production of secondary particles (inelastic collision). If the mean interaction length (mean free path) is $\lambda_{\text {int }}(\rho)$, for ultra-relativistic particles the rate of interaction is $r_{\text {int }}(\rho)=c / \lambda_{\text {int }}(\rho)$. If the mean inelasticity, i.e. average fraction of energy lost per interaction, is $\bar{\alpha}(\rho)$ then the effective loss rate for these non-continuous losses is $r_{\text {loss }}^{\text {(int) }}(\rho)=\bar{\alpha}(\rho) r_{\text {int }}(\rho)$. Of course, if $\bar{\alpha}(\rho) \ll 1$ then one can approximate the interactions as continuous energy losses. 
In the Monte Carlo simulations Bethe-Heitler pair production was treated as a continuous loss processes, but hadronic collisions of protons and neutrons as well as photo-disintegration of nuclei were treated by the Monte Carlo method. Neutrons produced, e.g. in $p \gamma \rightarrow n \pi^{+}$or during photo-disintegration of nuclei $\left(N \gamma \rightarrow N^{\prime}+i n+j p\right.$, where $N$ and $N^{\prime}$ are the parent and daughter nuclei, and $i$ and $j$ are integers), may decay inside the box into protons which continue to be accelerated, or they may escape from the box to decay outside into cosmic rays depending on the neutron's Lorentz factor and the physical dimensions of the acceleration region. Since the simulations are for a given $\rho_{\text {cut }}^{(p)}$, $\rho_{\max }$ and $\delta$, and the size of the box depends on the (unknown) normalization of the diffusion coefficients, we have performed simulations separately for the two extreme cases.

In the simulation with $N_{0}$ particles injected, a particle is injected at time $t=0$ with rigidity $\rho(0)=\rho_{0}$ and statistical weight $w(0)=w_{0}=1 / N_{0}$. Its subsequent rigidity, $\rho(t)$, and weight, $w(t)$, are determined after successive time steps $\Delta t$ chosen to be much smaller than the smallest time-scale in the problem. In each time step, first the rigidity is changed, $\rho(t+\Delta t)=\rho(t)\left[1+\Delta t r_{\text {acc }}(\rho)\right]$, and then the probability of escaping in time $\Delta t$ is estimated as $P_{\text {esc }}=\left\{1-\exp \left[-\Delta t r_{\text {esc }}(\rho)\right]\right\}$. Then $w(t) P_{\text {esc }}$ particles with energy $E$ are binned in the histogram of accelerated particles for the particle's species, and the particle's weight is changed to reflect the fraction not escaping, $w(t+\Delta t)=w(t)\left(1-P_{\text {esc }}\right)$.

Next, the probability of interacting in time $\Delta t$ is estimated, $P_{\text {int }, A, Z}=\left\{1-\exp \left[-\Delta t r_{\text {int }}(\rho, A, Z)\right]\right\}$, and a random number is generated to determine whether or not an interaction takes place. If an interaction does take place, the energy of the target photon in the proton or nucleus rest frame (NRF), $\epsilon^{\prime}$, is sampled from

$p\left(\epsilon^{\prime}\right) \propto n\left(\epsilon^{\prime}\right) \sigma\left(\epsilon^{\prime}\right) \quad$ for $\epsilon_{\mathrm{thr}}^{\prime}<\epsilon^{\prime}<2 \gamma \epsilon_{\max }$

where $n(\epsilon) d \epsilon$ is the LAB-frame number density of target photons with energy in the range $\epsilon$ to $(\epsilon+d \epsilon), \sigma\left(\epsilon^{\prime}\right)$ is the relevant cross section in the NRF, $\epsilon_{\text {thr }}^{\prime}$ is the NRF threshold energy for the process considered, $\gamma$ is the Lorentz factor of the proton or nucleus, and $\epsilon_{\max }$ is maximum target photon energy in the LAB frame. For ultra-relativistic projectiles, the photon's direction in the NRF will be anti-parallel to the LAB-frame direction of the projectile. In the nucleon case (either a primary proton or a nucleon resulting from photo-disintegration), pion photoproduction interactions are simulated in the the NRF using the SOPHIA event generator, and the 4-momenta of all the produced particles are Lorentz transformed to the LAB frame. If the leading nucleon remains a proton, its rigidity ( $\rho_{i}$ before the interaction) is changed to $\rho=\rho_{i}(1-\alpha)$, where $\alpha$ is the inelasticity for that collision. However, if it is a neutron we consider two extreme possibilities as discussed earlier: (a) it decays to a proton inside the acceleration zone and will continue to be accelerated; or (b) it escapes from the acceleration zone, decaying outside and adding to the pool of cosmic rays which have escaped from the accelerator. Escaping protons, neutrons, nuclei and neutrinos are binned in energy.

In the case of interactions of nuclei, the number of emitted nucleons and $\alpha$ particles is determined according to branching ratios of the various interaction channels. Whenever the BR process is chosen, we check whether or not neutrinos are produced and calculate their energy according to the $\Delta$ resonance kinematics (see Allard et al. 2006 for more details)

Next we take account of the additional escape resulting from the rigidity decreasing during the interaction. This is more important for pion photoproduction losses than for photodisintegration in which the rigidity does not necessarily decrease. The probability of the particle immediately escaping downstream due to falling out of the box is estimated,

Prob. (escape, $\left.\rho_{i} \rightarrow \rho\right)=\frac{L_{2}\left(\rho_{i}\right)-L_{2}(\rho)}{L\left(\rho_{i}\right)}=\left[1-\left(\frac{\rho}{\rho_{i}}\right)^{\delta}\right] \ell_{2}$,

and a random number is generated to determine if this happens. If the particle does escape, then $w(t+\Delta t)$ particles with momenta $\rho$ are binned in a histogram of accelerated particles, and a new particle is injected with rigidity $\rho_{0}$ and weight $w_{0}$. If the particle does not escape, then the particle's rigidity and weight are evolved through a new time step as described above. Finally, if the time since injection exceeds a maximum time which may represent the time during which the accelerator is active, $t_{\text {active }}$, the acceleration ceases and a new particle is injected. In most cases we set to $t_{\text {active }}=10 \mathrm{Gyr}$, but we shall explore shorter activity time scales.

We note that the term $r_{\text {loss }}\left(\rho_{\text {cut }}^{(p)}, Z=1\right)$, used to normalize the rigidity dependence of the acceleration rate, represents the loss rate of a proton at a its cut-off rigidity $\rho_{\text {cut }}^{(p)}$ in the extragalactic photon background (i.e. the CMB and the inergalactic IR/opt/UV background). Using the parameter $\rho_{\text {cut }}^{(p)}$ (i.e. the rigidity at which the loss rate would equal the acceleration rate for protons in an accelerator "bathed" in the intergalactic photon background) as a reference will turn out to be very convenient for estimating the effect of different physical parameters $\left(\rho_{\max }\right.$, $\delta$ and the scaling factor of the IR/opt/UV background) on the spectrum of accelerated protons and nuclei. Our Monte Carlo results and the conclusions of our study will be presented in the following two sections.

\section{Results}

In this section, we describe the results of our calculations for different combinations of the above-mentioned acceleration parameters. For each combination of $\delta, \rho_{\max }$ and the scaling of the IR/opt/UV, we calculate the expected output spectrum for different values of $\rho_{\text {cut }}^{(p)}\left(10^{20}, 10^{21}, 10^{22} \mathrm{~V}\right)$ and various compositions injected at the shock: (i) pure proton; (ii) pure iron; and (iii) a mixed composition similar to low energy Galactic cosmic rays (see Allard et al. 2005, and references therein). In Figs. 6-11 we give the spectra of nuclei, showing contributions of different groups of elements, accelerated such that in the absence of interactions and losses the spectra would be proportional to $\rho^{-\Gamma}$, and in the present work we set the nominal spectral index to be $\Gamma=2$. The choice of other reasonable values of $\Gamma$ would make only a minor difference to the relative compositions of accelerated particles, and the relative contributions of secondary nucleons, photons, and neutrinos would be somewhat higher (lower) for harder (softer) spectra, but this would not modify the main conclusions of the present work. In these figures we also show the flux of neutrinos plus antineutrinos (summed over all flavors) produced during acceleration, and for the case where neutrons are assumed not to decay inside the acceleration zone we give the contribution to the total escaping cosmic rays of neutrons produced during pion photoproduction and photo-disintegration of nuclei. Note that unless otherwise specified we assume that the source is active for $10 \mathrm{Gyr}$, and that the injection rate does not depend on time.

Figure 6 shows the accelerated cosmic-ray spectra assuming $\rho_{\max }=10^{25} \mathrm{~V}$ (corresponding to an infinite shock), Kolmogorov turbulence (diffusion coefficient rigidity-dependence index $\delta=$ $1 / 3$ ), a photon background equivalent to the extragalactic background, and neutrons decaying inside the acceleration zone. For 


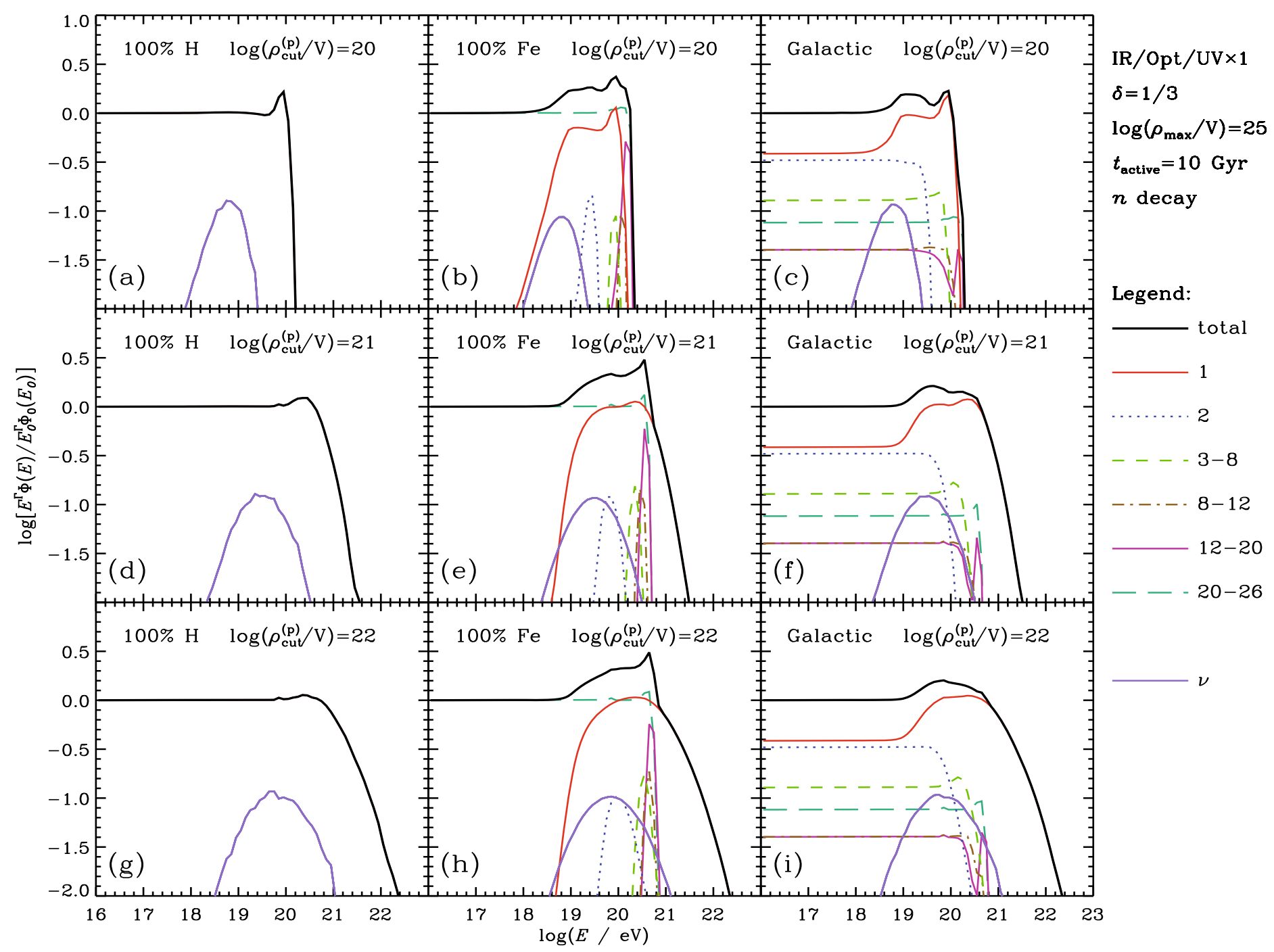

Fig. 6. Spectra of the various nuclei emerging after acceleration together with the spectra of neutrons and neutrinos (sum of all flavors) escaping from the acceleration region. The initial composition is $100 \% \mathrm{H}$ (left column), $100 \% \mathrm{Fe}$ (middle column), mixed composition representative of that observed in Galactic cosmic rays (right column). The acceleration rate is such that $\rho_{\text {cut }}^{(p)}=10^{20} \mathrm{~V}$ (top row), $10^{21} \mathrm{~V}\left(\right.$ middle row), and $10^{22} \mathrm{~V}$ (bottom row). The IR/Opt/UV background is taken to be that at $z=0$, neutrons are assumed to decay inside the acceleration zone, $\kappa(\rho) \propto \rho^{1 / 3}$ and $\rho_{\max }=10^{25}$ V. Other acceleration parameters are as indicated. Curves are for atomic numbers as indicated in the legend, with the topmost solid curve being the total spectrum, middle solid curve being $Z=1$, and the bottom solid curve being for the total neutrino spectrum.

this case, the acceleration process is interaction-limited because $\rho_{\max }$ is extremely high, and we see that there is no obvious scaling of the maximum energies of the various species with their mass or charge - this is consistent with our expectation based on Figs. 3 and 5. Indeed, although the interaction threshold for a given photon background will occur at energies energies more or less proportional to the mass number of nuclei, such a scaling is not observed for the various cases studied in Fig. 6. This is because the mean free paths also scale with the mass, which means that the energy at which the photo-disintegration mean free path becomes of the same order as the acceleration length scales in a non-trivial way with mass number $A$. However, heavy nuclei can reach higher energies than light and intermediate nuclei due to their higher interaction energy threshold and higher acceleration rate at a given energy.

Once the acceleration rate becomes lower than the interaction rate for a given component, a sharp cut-off takes place as the nucleus changes to a lower mass and lower energy. As can be seen in Figs. 3 and 5, the cut-off energy depends on $\rho_{\text {cut }}^{(p)}$ and $\delta$ (see below). Due to the very low values of the interaction length for nuclei with $\mathrm{CMB}$ photons, such cut-offs seem to be extremely difficult to avoid even for very rapid acceleration (corresponding to high values of $\rho_{\text {cut }}^{(p)}$ ). The proton component also experiences a cut-off at energies depending on $\rho_{\text {cut }}^{(p)}$ because of the increase of the photopion interactions loss rate with energy and the subsequent possibility of protons to fall out of the box, the latter being more important for protons than for nuclei because nuclei mainly lose energy by ejecting nucleons. This cut-off is however smoother than in the case of nuclei due the different nature of the energy losses. In the case we study here, i.e. acceleration not limited by confinement and a source active during the Hubble time, protons can reach an energy slightly higher than heavy nuclei, their lower acceleration rate (at a given energy) being compensated for by a lower interaction rate. When the acceleration rate is high enough (e.g. $\rho_{\text {cut }}^{(p)}>10^{21} \mathrm{eV}$ ) most species of nuclei (even the lightest) can reach energies above $10^{20} \mathrm{eV}$.

For the case of a mixed composition or $100 \%$ iron there is a bump in the spectrum at the highest energies due to secondary 


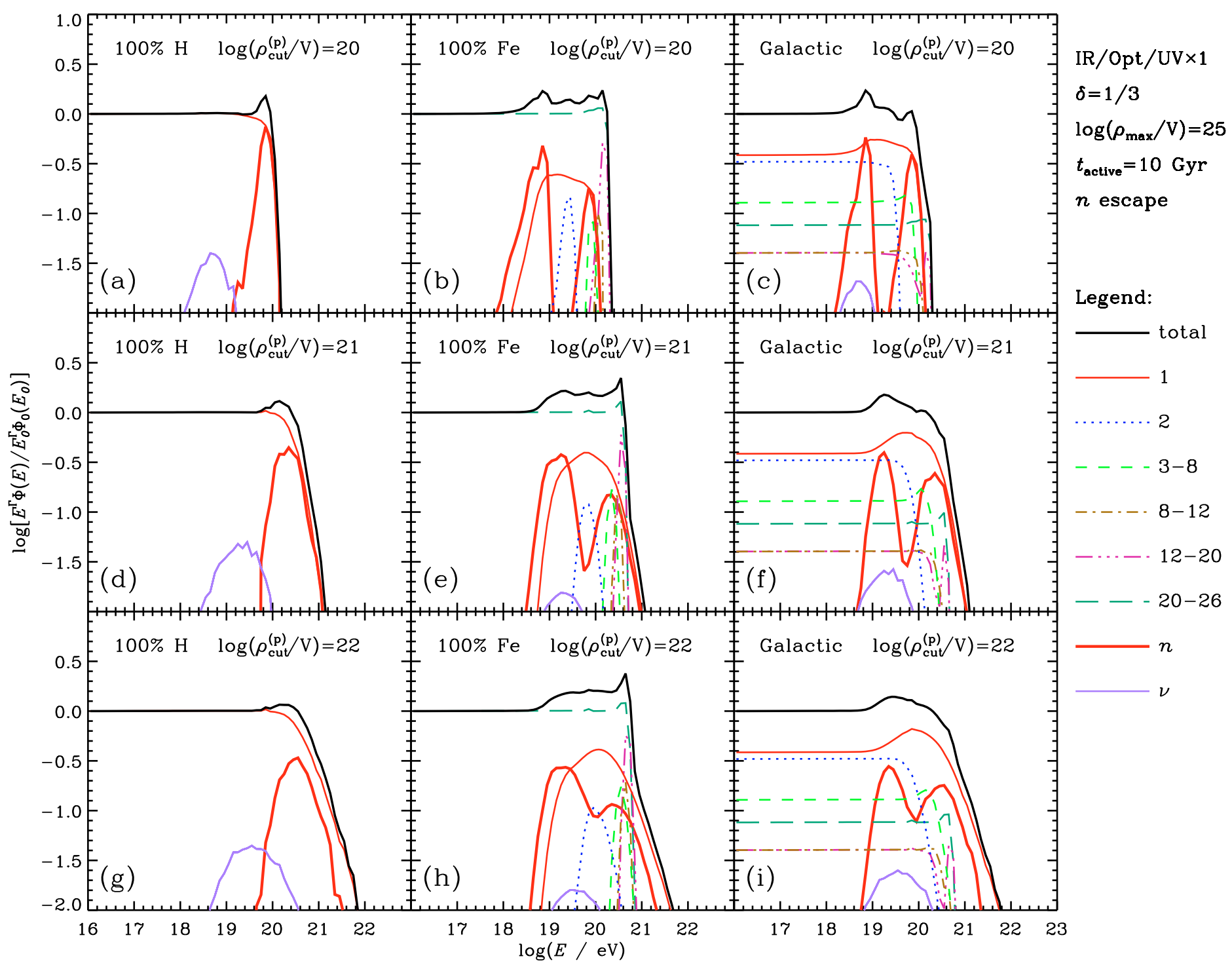

Fig. 7. As Fig. 6 except that neutrons are assumed to rapidly escape from the acceleration zone where they decay into protons. Curves are as in Fig. 6 except that the additional thick solid curve shows the contribution of neutrons.

nucleons, and it starts quite abruptly at the energy at which the photodisintegration interaction rate becomes higher than the acceleration rate. Secondary protons and protons from decay of secondary neutrons in the box as assumed Fig. 6 continue to be accelerated and produce a tail in the energy spectrum at the highest energies.

The alternative case in which neutrons escape before decaying is equivalent to adding a supplementary escape term as approximately half of the produced secondary nucleons leave the box without being further accelerated. In this case, protons also have an additional probability of escaping after each pion photoproduction interaction equivalent to the probability of isospin flip (see Mücke et al. 2000). As a consequence, the cut-off in the proton component in Fig. 7 is sharper than for the case of neutron decay inside the box shown in Fig. 6. In the 100\% proton case there is a bump in the spectrum due to neutrons which starts at the energy at which the pion photoproduction interaction rate becomes higher than the gain rate for a given value of $\rho_{\text {cut }}^{(p)}$. When nuclei are injected there is also a second bump at lower energies corresponding to the interaction threshold for photo-disintegration.

\section{Case of a high ambient photon background}

As mentioned earlier, the photon background in astrophysical sources could be significantly higher than in intergalactic space, and to mimic such stronger fields we have scaled the IR/opt/UV intergalactic background upward by various factors to investigate the influence of the photon density on the accelerated spectrum and composition. We have kept the other parameters the same as for Fig. 6, i.e. $\delta=1 / 3, \rho_{\max }=10^{25} \mathrm{~V}$ and neutrons decaying inside the box, and show in Fig. 8 the result for an IR/opt/UV background 100 times higher than in intergalactic space. As can be seen in Fig. 1, such a background is comparable to the background expected in radio lobes located $\sim 200 \mathrm{kpc}$ from a relatively powerful FSRQ and so can serve as a typical reference for what can be expected in some classes of source (see discussion below). 


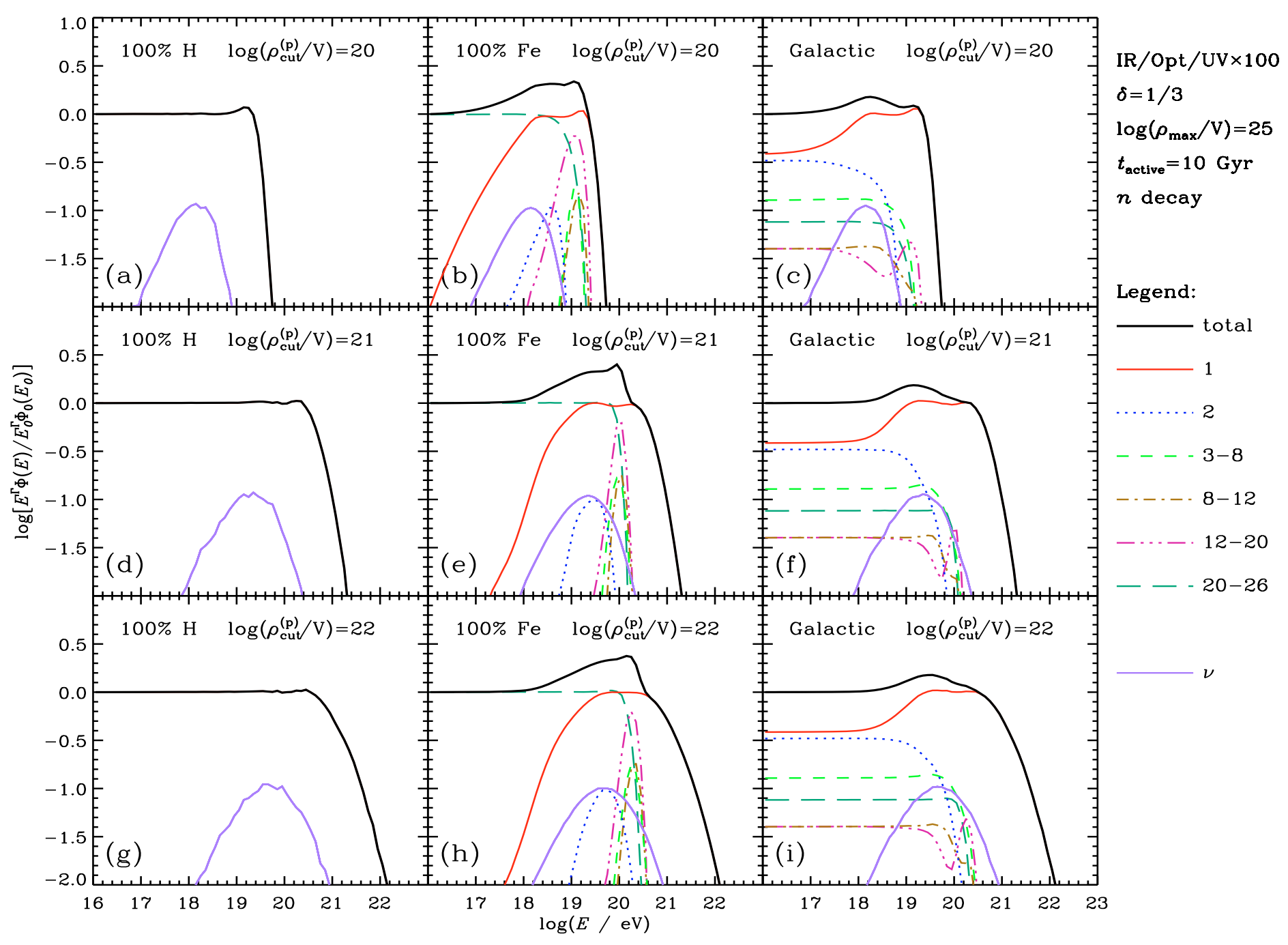

Fig. 8. As Fig. 6 except that the IR/Opt/UV is taken to be 100 times that at $z=0$.

Figures 3 and 5 show that such a high background will lower the energy at which the spectrum of a particular species cuts off as the balance between acceleration and energy losses/photodisintegration losses occurs at a lower energy. We see in Fig. 8 that this effect is stronger for nuclei than protons, but that nevertheless most species can still be accelerated well above $10^{19} \mathrm{eV}$. We have found that this applies even for IR/opt/UV backgrounds 1000 times higher than in the extragalactic space, but that when IR/opt/UV background is $10^{4}$ times higher than in extragalactic space protons and nuclei are not accelerated above $10^{19} \mathrm{eV}$ whatever the value of $\rho_{\text {cut }}^{(p)}$.

High photon backgrounds present at sources are usually thought to eliminate nuclei during the acceleration process giving rise to a $100 \%$ proton composition. However, we find this not to be the case for any combination of the acceleration parameters that we have explored.

\section{Influence of rigidity dependence of the diffusion coefficient}

The energy dependence of the acceleration rate depends on the rigidity dependence of the diffusion coefficient $\kappa \propto \rho^{\delta}$ in the vicinity of the shock. As can be seen in Figs. 3 and 5, the powerlaw index $\delta$ affects the competition between acceleration and energy losses. We show in Fig. 9 results for $\delta=1$ (Bohm scaling) while keeping all other parameters the same as used for Fig. 8 for which $\delta=1 / 3$ (Kolmogorov turbulence) was used. We see that as result of the steeper dependence of the acceleration rate on energy the cut-offs in the spectra of the various nuclear species are shifted to higher energies with the gap between protons and nuclei being reduced, and heavy nuclei being able to reach as high energies as protons even with the IR/Opt/UV background 100 times that in intergalactic space.

We introduce here Fig. 10 to illustrate some of the effects and dependencies that we have noted already, and to highlight them in a form which more clearly shows their origin. The effect of changing the rigidity dependence of the acceleration rate noted above is even more pronounced if neutrons escape from the acceleration zone (see Fig. 10f). The comparison between Figs. 10d,e shows there to be a dramatic increase in the maximum energies of protons and nuclei in a very high radiation field (a factor $10^{4}$ increase in IR/Opt/UV) when the rigidity dependence of the diffusion coefficient is changed from $\rho^{1 / 3}$ (Kolmogorov) to $\rho^{1}$ (Bohm).

Another consequence of a stronger dependence of the acceleration rate on $\rho$ is a sharper cut-off of the proton component because the gain rate more rapidly decreases above $\rho_{\text {cut }}^{(p)}$ and it is then very difficult to continue to accelerate protons once they 


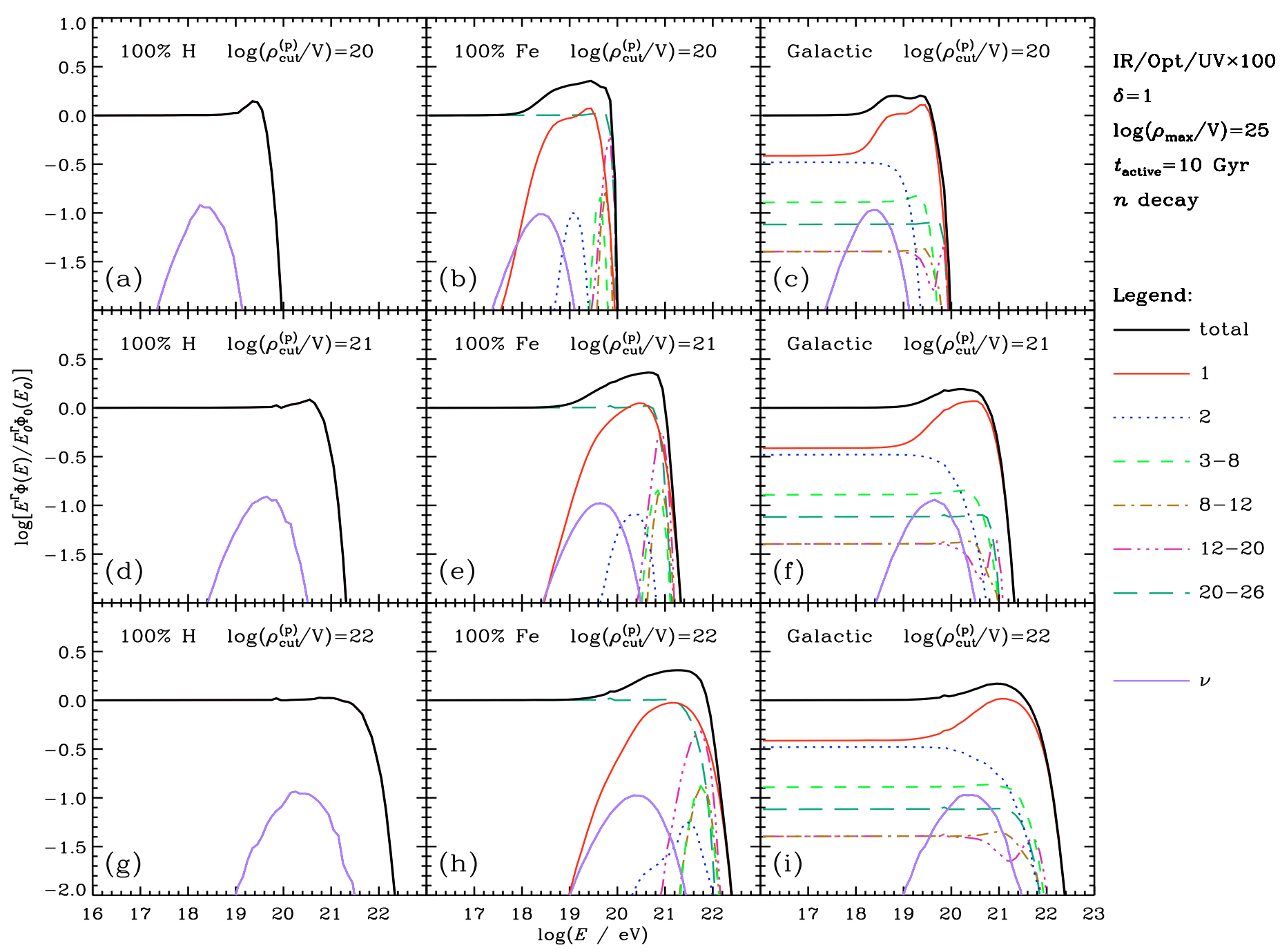

Fig. 9. As Fig. 8 except that $\kappa(\rho) \propto \rho^{1}$.

start to interact, even if they do not escape from the source immediately.

\section{Confinement-limited acceleration}

So far we have only considered cases where the acceleration is limited by interactions which we model by setting $\rho_{\max }$ to a very high value, $10^{25} \mathrm{~V}$. However, astrophysical sources are of finite size and so are expected also to have limited capability of confining high rigidity cosmic rays, e.g. when their larmor radius becomes comparable to the size of the acceleration medium. Confinement-limited acceleration processes can be modeled by lowering the value of $\rho_{\max }$. Figure 11 shows the result of our calculations assuming $\rho_{\max }=10^{19} \mathrm{~V}$ while keeping all the other parameters the same as in Fig. 8 (neutrons decay inside the box, $\delta=1 / 3$ and a scaling factor of 100 for the IR/opt/UV background). In all cases, we see that the proton component cuts off around $10^{19} \mathrm{eV}$ due to the confinement limitation. With a rigidity cut-off one would expect that in absence of energy losses the cut-off energies would be proportional to the charge for the various species, but one can see in Fig. 11 that for this choice of the parameters this scaling only applies very crudely and only for the rapid acceleration case $\left(\rho_{\text {cut }}^{(p)}=10^{22} \mathrm{eV}\right)$. The acceleration of nuclei still appears to be interaction-limited, but nevertheless heavy nuclei are always accelerated above $10^{20} \mathrm{eV}$ when $\rho_{\text {cut }}^{(p)} \geq 10^{21} \mathrm{eV}$ and are accelerated to higher energies than the proton component.

We find that for $\rho_{\max }=10^{19} \mathrm{~V}$ provided there is a steep energy dependence of the rigidity gain rate $(\delta=1)$ the expected scaling of the cut-off energies with $Z$ applies for the case of a photon background with the IR/Opt/UV 100 times that in intergalactic space (top panel of Fig. 10) and even with the IR/Opt/UV 1000 times higher (Fig. 10g). The expected scaling also applies for lower photon backgrounds,

For higher values of $\rho_{\max }$, e.g. $10^{21} \mathrm{~V}$, there will be very little difference for nuclei to the $\rho_{\max }=10^{25} \mathrm{~V}$ case (e.g. $\rho_{\text {cut }}^{(p)}=10^{21} \mathrm{~V}$ and $10^{22} \mathrm{~V}$ in Fig. 8) as interactions already cut off their spectra at rigidities below $Z \times 10^{21} \mathrm{eV}$. Only the proton spectrum will be confinement-limited reducing the gap between the cut-off energies of protons and nuclei.

\section{Influence of duration of source activity}

Some of the astrophysical sources that are candidates for the acceleration of cosmic-rays to the highest energies, e.g. FSRQ which according to unification schemes (e.g. Urry \& Padovani 1992) are the beamed counterparts of the powerful FanaroffRiley Class II (FR-II) radio galaxies, are transitory sources with 


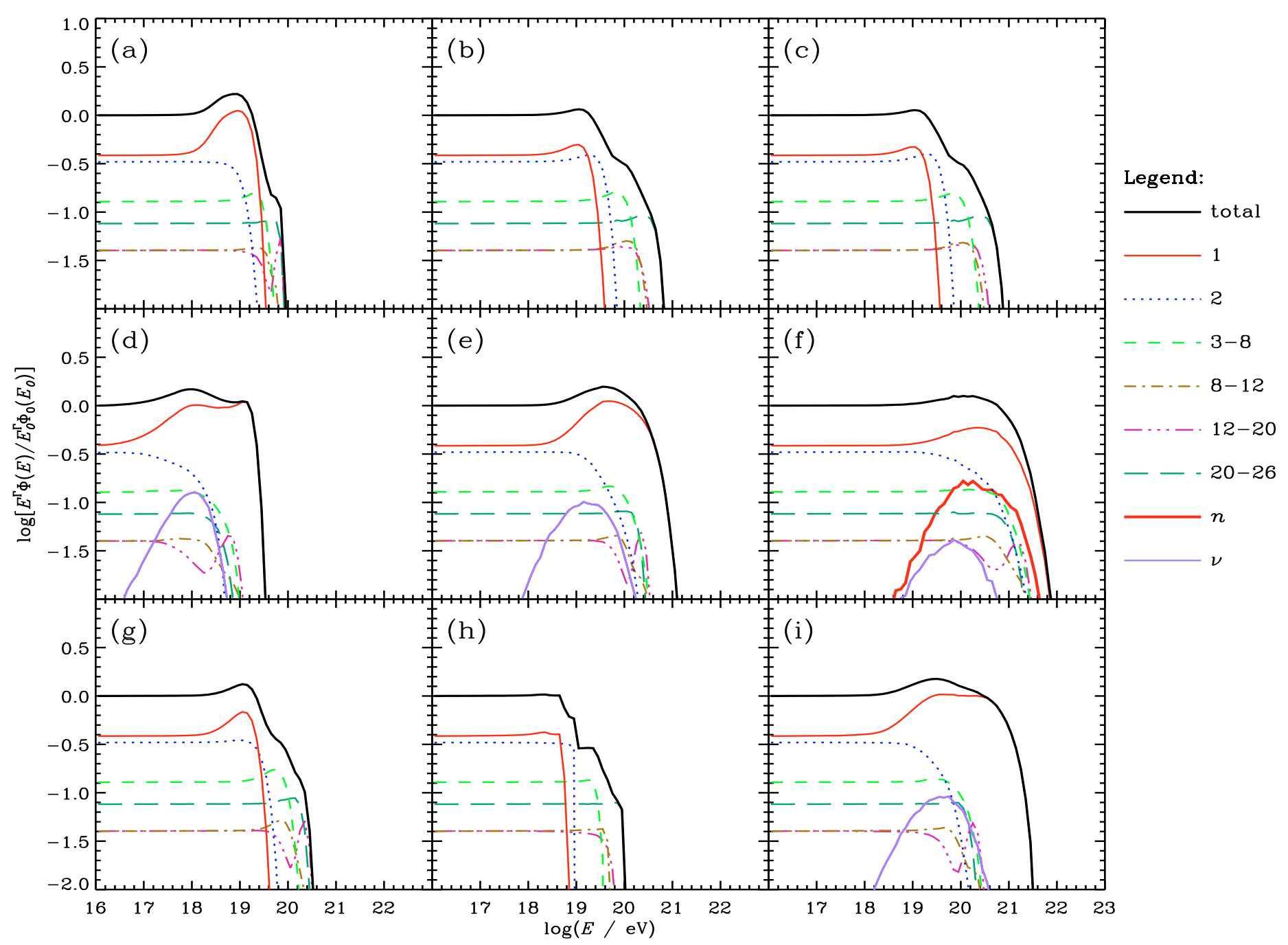

Fig. 10. Miscellaneous combinations of parameters: a), b) and c). As Fig. 6 except that $\delta=1, \rho_{\max }=10^{19} \mathrm{~V}$ and only the mixed composition is shown. d) Mixed composition, $\rho_{\text {cut }}^{(p)}=10^{22} \mathrm{~V}$, IR/Opt/UV $\times 10000, \delta=1 / 3, \rho_{\max }=10^{25} \mathrm{~V}$ and neutrons decay inside the box. e) Same as d) but with $\delta=1$. f) Mixed composition, $\rho_{\text {cut }}^{(p)}=10^{22} \mathrm{~V}$, IR/Opt $/ \mathrm{UV} \times 1000, \delta=1, \rho_{\max }=10^{25} \mathrm{~V}$ and neutrons escape from the box. g) Same as c) but with IR/Opt/UV $\times 10000$. h) Same as Fig. 8 for the mixed composition and $\rho_{\text {cut }}^{(p)}=10^{22} \mathrm{~V}$ except that the source activity duration is 10 Myr. i) Same as $\mathbf{h}$ ) but for a source activity duration of $100 \mathrm{Myr}$.

a life time small compared to the age of the universe. The finite duration of the source activity (by activity we mean the period during which the source is able to accelerate cosmic-rays) does not necessarily impact on the spectrum and composition of accelerated cosmic rays. Indeed, if the acceleration time scale is small compared to the duration of source activity, there will essentially be no difference. An example is shown in Fig. 10i, where the source parameters of Fig. 8 are used except for the source activity duration which is only $100 \mathrm{Myr}$. We see that for a high acceleration rate $\left(\rho_{\text {cut }}^{(p)}=10^{22} \mathrm{~V}\right)$ the output is essentially identical to that shown in Fig. 8, with the nuclear components being almost indistinguishable between the two cases and the only major difference being that the high energy tail of the proton component is absent when the source has the shorter lifetime.

The situation changes when the source activity duration is less than the time needed to accelerate protons at the highest energies. Fig. 10h shows the spectrum and composition for the same parameters as Fig. 10i except that the source activity duration is only $10 \mathrm{Myr}$. In this case, the proton component is even more affected due to its lower acceleration rate and the maximum energies scale proportionally to the charge of the species. In fact the proton cut-off rigidity, at $6 \times 10^{18} \mathrm{~V}$, is significantly lower than $10^{20} \mathrm{~V}$ which would naively be expected based on reading off $\rho$ in Fig. 3 at $x=3 \mathrm{Mpc}$ (corresponding to $10 \mathrm{Myr}$ ) for the $\rho_{\text {cut }}^{(p)}=10^{22} \mathrm{~V}$ and $x_{\text {gain }} \propto \rho^{1 / 3}$ case. The reason for this is that for injection of a proton at $\rho=\rho_{0} \ll \rho_{\text {cut }}^{(p)}$ at time $t=0$, and acceleration for time $t=x / c$, the proton has a rigidity of $\rho_{\max } \approx\left[x \delta / x_{\text {gain }}\left(\rho_{\text {cut }}^{(p)}\right)\right]^{1 / \delta} \rho_{\text {cut }}^{(p)}$. Once more, the heaviest components are close to the default case of Fig. 8 because of their higher charge (and acceleration rate), even for such a short acceleration time.

\section{Conclusions}

We have studied DSA of protons and nuclei in astrophysical sources in the presence of background radiation fields and there are some general conclusions we can draw. Provided the ambient magnetic field is not too high (see Fig. 4 for protons) we find that when the acceleration mechanism is not limited by confinement protons usually reach higher energies than nuclei which 


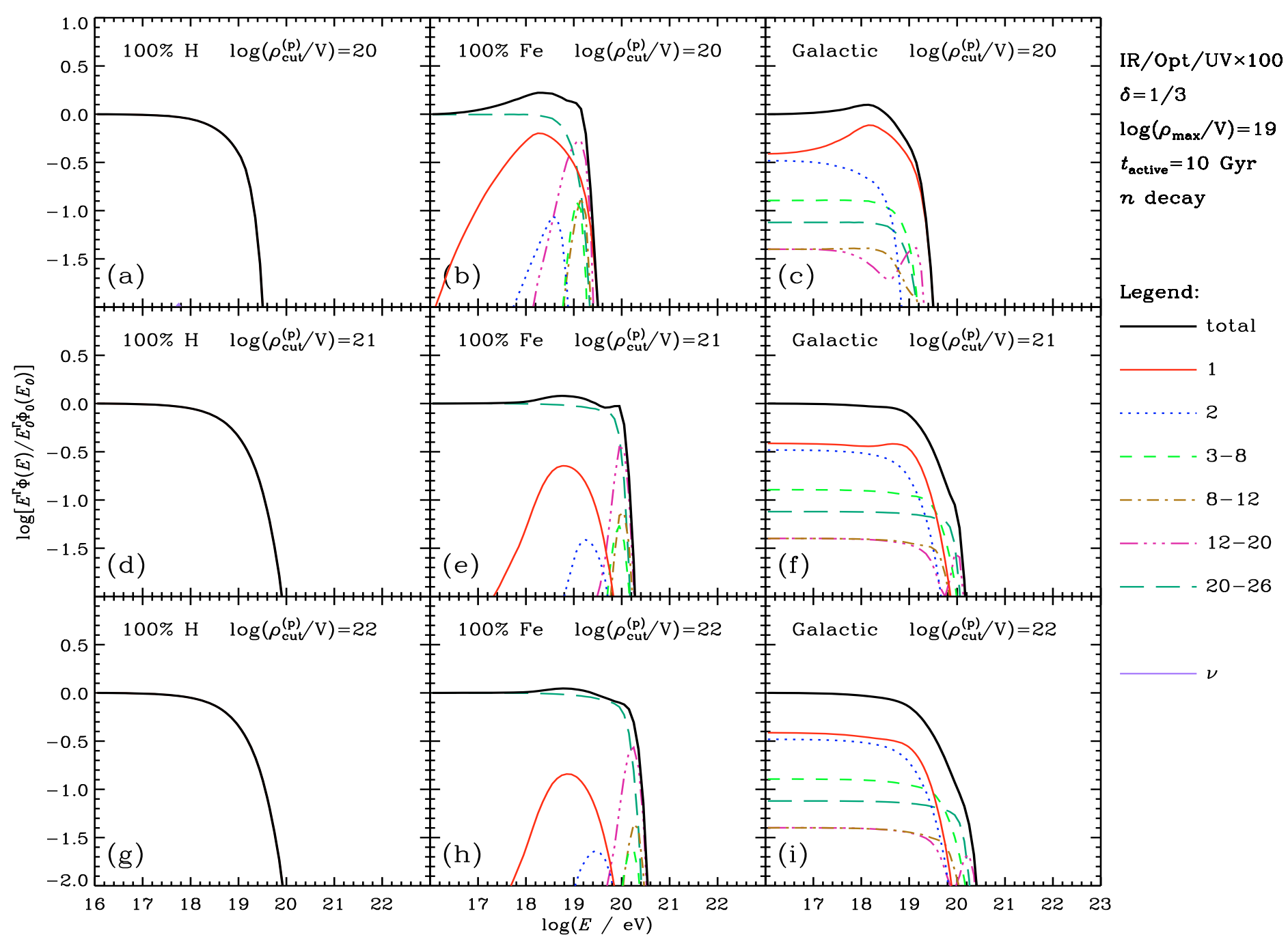

Fig. 11. As Fig. 6 except that $\rho_{\max }=10^{19} \mathrm{~V}$.

are more limited by interactions. This conclusion is particularly true for the conservative assumption that the turbulence in the vicinity of the shock is of Kolmogorov-type (i.e. a slow dependence of the acceleration rate with energy). If the turbulence brings the diffusion coefficient dependence closer to the Bohm regime we find that heavy nuclei can usually reach maximum energies as high as those of protons, even in the case of very high ambient photon fields. Whenever the acceleration mechanism is not confinement-limited we find there to be no trivial scaling of the maximum energies of the various species with their mass or atomic number.

It is beyond the scope of the present paper to calculate the expected spectra at Earth for each source model, and we refer the reader to Allard et al. (2005-2008) for detailed studies of the propagation of cosmic-ray nuclei in the extragalactic radiation fields. However, the non-trivial scaling of the maximum energy with the mass or atomic number should not affect significantly the results obtained in propagation studies in which maximum energies are usually assumed to be proportional to the charge. Indeed, we have seen that secondary nucleons produced in interactions within the accelerator continue to be accelerated (except for escaping neutrons) and can then reach higher energies. On the other hand, nuclei at energies above $\sim A \times\left(4 \times 10^{18} \mathrm{eV}\right)$ are expected to be almost fully photo-disintegrated by $\mathrm{CMB}$ photons during propagation (see Allard et al. 2008), and this means that nuclei accelerated above these energies would not be seen at Earth and would simply release their nucleons at energies a factor $A$ lower. Whether or not nuclei above $\sim A \times 4 \times 10^{18} \mathrm{eV}$ are photo-disintegrated within the source or during the propagation will make little difference to what is observed at Earth. Furthermore, any bumps in the spectrum due to secondary nucleons should be smoothed by source to source variability as well as by cosmological effects during the propagation. Bumps in the cosmic ray spectrum would remain after propagation of cosmic-rays from a single source, but not remain after summing the contributions of all sources in the Universe because of the large range of propagation times from the sources to the earth except for the case of a dominant contribution by a very nearby source.

One important point to emphasize is that even in the presence of strong photon backgrounds, nuclei are accelerated to energies above $10^{19} \mathrm{eV}$ whenever protons are, even though nuclei are more limited by interactions than nucleons. Hence, our results show that DSA at sources with physical conditions that would cause nuclei to be fully photodisintegrated during acceleration but allow protons to reach energies above $10^{20} \mathrm{eV}$ are excluded. In other words a pure proton composition is unlikely to result from an acceleration process whenever a mixed 
composition is injected at a shock. A $100 \%$ proton composition would therefore require a different explanation such as injection of only protons at the shock, or propagation in a medium optically thick to photodisintegration of nuclei in the vicinity of the source.

It could be argued that our modeling of the photon background in which we scaled the extragalactic IR/Opt/UV by factors between 1 and 10000 is too simplistic. However, we shall now consider our results briefly in the context of acceleration in the radiation field at a shock at a hot-spot of an FR-II radio galaxy. FR-II radio galaxies are thought to be the UNbeamed counterparts of FSRQ. Given the spectral energy distribution (SED) of a "typical" FSRQ, we may infer the radiation field at the likely diffusive shock acceleration sites at the far end of an FR-II jet. In Fig. 1 we showed the radiation field expected at $200 \mathrm{kpc}$ along the jet from 0208-512 based on the SED for this FSRQ given in Fig. 5 of Tavecchio et al. (2002), and compared it with the IR/Opt/UV at $z=0$, and IR/Opt/UV fields that are 100 and 1000 times higher. We see that while the low energy part of the SED is important, the high energy part of the SED, i.e. X-rays and above, will have little effect as target photons for pion photoproduction or photo-disintegration of nuclei. However, there will actually be a very large range in the normalizations of the FSRQ blazar field at the hot-spot for two reasons. First, the distance from the core to the hot-spots for FR-II is probably in the range $50-800 \mathrm{kpc}$, giving rise to a range of a factor of about 300 in radiation density. Secondly, there is also a large range in luminosity of FSRQ - Zhou et al. (2007) found that $\log \left[L_{v}(1.5 \mathrm{GHz}) / \mathrm{W} \mathrm{Hz}^{-1}\right]$ to range from 26.4 to 29.0 with a mean value 27.7 for 45 FSRQ. This is to be compared with that of $0208-512$ for which $\log \left[L_{v}(1.5 \mathrm{GHz})\right] \approx 28.5 \mathrm{~W} \mathrm{~Hz}^{-1}$. Nevertheless, as the shape of the SED of the extragalactic of photon background is not very different from the FSRQ SED shown in Fig. 1 our results can then give some hint of the influence of the photon backgrounds in this type of source. However, we plan to consider more specifically the acceleration of cosmic-rays in FR-II galaxies in a future paper.

Interaction-limited acceleration processes are also expected to result in production of gamma-rays and neutrinos. As shown by Protheroe (2004), the position of the neutrino peak can help to constrain the acceleration parameters in proton acceleration sources and the characteristics of the source. However, as can be seen in the various figures in Sect. 4, when we include nuclei neutrino fluxes cannot give any strong constraints on the injected composition at the shocks.

When the size of the source becomes comparable to or smaller than the Larmor radius of protons at the highest energies, the acceleration becomes limited by confinement, in which case heavy nuclei are accelerated to higher energies than protons unless the acceleration is very slow and/or the photon background is very high. In the case of the maximum proton energy being below $10^{20} \mathrm{eV}$ the maximum energies of all species are proportional to their charge, even in the presence of high photon backgrounds, provided the acceleration parameters are favorable $\left(\rho_{\text {cut }}^{(p)} \geq 10^{21} \mathrm{eV}\right.$ and $\delta$ close to "Bohm scaling"). Such a composition is especially interesting in the context of recent cosmic-ray data. Indeed, preliminary results from the Pierre Auger Collaboration show the composition possibly getting heavier above $10^{19} \mathrm{eV}$ (see Unger et al. 2007). If this trend is confirmed, the most likely explanation would involve confinement-limited acceleration with the maximum energy of the species scaling with the charge. A composition getting heavier above $10^{19} \mathrm{eV}$ is indeed very difficult to obtain with propagation effects only (see Allard et al. 2005-2008) when protons are accelerated up to the highest energies. We have also seen in previous sections that in most cases if the acceleration is interaction-limited then nuclei are not accelerated to higher energies than protons. Interestingly, it has been shown recently (Allard et al. 2008) that the highest energy cosmic-ray spectrum can be successfully fitted by assuming a low proton maximum energy $\left(\sim 10^{19} \mathrm{eV}\right)$ and a charge-scaling maximum energy for the other species, providing abundances of heavy nuclei slightly higher than in the Galactic cosmic rays. Although other possibilities are not ruled out, such a scenario where protons are not required to be accelerated above $10^{20} \mathrm{eV}$ but where the highest energy cosmic-rays are provided by the heavy component would completely change the expectations and constraints on possible accelerators like blazars (Mücke et al. 2003) or radiogalaxies. We shall investigate these implications in forthcoming papers. Finally, we note that confinement-limited acceleration is unlikely to provide strong neutrino fluxes at high energy.

Acknowledgements. This work was supported in part by the Australian Research Council through Discovery Project grant DP0881006 and by the AustraliaFrance Cooperation Fund in Astronomy.

\section{References}

Allard, D., Parizot, E., Olinto, A. V., Khan, E., \& Goriely, S. 2005, A\&A, 443, L29

Allard, D., Ave, M., Busca, N., et al. 2006, J. Cosmo. Astro-Part. Phys., 9, 5

Allard, D., Olinto, A. V., \& Parizot, E. 2007, A\&A, 473, 59

Allard, D., Busca, N. G., Decerprit, G., Olinto, A. V., \& Parizot, E. 2008, J. Cosmo. Astro-Part. Phys., 10, 33

Benford, G., \& Protheroe, R. J. 2008, MNRAS, 383, 663

Berezhko, E. G., \& Krymski, G. F. 1988, Usp. Fiz. Nauk, 154, 49

Berezinsky, V. S., \& Zatsepin, G. T. 1969, Phys. Lett., 28B, 423

Biermann,, P. L., \& Strittmatter, P. A. 1987, ApJ, 322, 643

Biermann, P. L., et al. 2008, proceedings for Origin, Mass, Composition and Acceleration Mechanisms of UHECRs (CRIS 2008) in Malfa (Salina Island - Italy), September 15-19 [arXiv: 0811.1848]

Blandford, R., \& Eichler, D. 1987, Phys. Rep., 154, 1

Drury, L. O'C. 1983, Space Sci. Rev., 36, 57

Drury, L. O'C., Duffy, P., Eichler, D., \& Mastichiadis, A. 1999, A\&A, 347, 370 Greisen, K. 1966, Phys. Rev. Lett., 16, 748

Hillas, A. M. 1984, A\&ARA, 22, 425

Inoue, S., Sigl, G., Miniati, F., \& Armengaud, E. 2007, Proceedings of the 30th ICRC, Merida, Mexico [arXiv: 0711.1027]

Jokipii, J. R. 1987, ApJ, 313, 842

Jones, F. C., \& Ellison, D. C. 1991, Space Sci. Rev. 58, 259

Kang, H., \& Jones, T. W. 2002, J. Kor. Astron. Soc., 35, 159

Khan, E., Goriely, S., Allard, D., et al. 2005, Astropart. Phys., 23, 191

Meyer, J. P., Drury, L. O’C., \& Ellison, D. 1997, ApJ, 487, 182

Mücke, A., Engel, R., Rachen, J. P., Protheroe, R. J., \& Stanev, T. 2000, Comp. Phys. Com., 124, 290

Mücke, A., Protheroe, R. J., Engel, R., Rachen, J. P., \& Stanev, T. 2003, Astropart. Phys., 18, 593

Norman, C. A., Melrose, D. B., \& Achterberg, A. 1995, ApJ, 454, 60

Padovani, P., \& Urry, C. M. 1992, ApJ, 387, 449

Protheroe, R. J. 1998, in Topics in cosmic-ray astrophysics, ed. M. A. DuVernois (New York: Nova Science Publishing), 2000, 258

Protheroe, R. J. 2004, Astropart. Phys., 21, 415

Protheroe, R. J., \& Stanev, T. 1999, Astropart. Phys., 10, 185

Protheroe, R. J., Donea, A.-C., \& Reimer, A. 2003, Astropart. Phys., 19, 559

Puget, J. L., Stecker, F. W., \& Bredekamp, J. H. 1976, ApJ, 205, 638

Rachen, J. P. 1996, Interaction processes and statistical properties of the propagation of cosmic-rays in photon backgrounds, Ph.D. Thesis, Bonn University Rachen, J. P., \& Biermann, P. L. 1993, A\&A, 272, 161

Schopper, R., Thorsten Birk, G., \& Lesch, H. 2002, Astropart. Phys., 17, 347

Stecker, F. W., Malkan, M. A., \& Scully, S. T. 2006, ApJ, 648, 774

Szabo, A. P., \& Protheroe, R. J. 1994, Astropart. Phys., 2, 375

Tavecchio, F., Maraschi, L., Ghisellini, G., et al. 2002, ApJ, 575, 137

Unger, M., Engel, R., Schüssler, F., Ulrich, R., \& Pierre AUGER Collaboration 2007, Astron. Nachr., 328, 614

Waxman, E. 2006, Nucl. Phys. B Proc. Suppl., 151, 46

Zatsepin, G. T., \& Kuzmin, V. A. 1966, Sov. Phys. JETP Lett., 4, 78

Zhou, J.-L., Fan, J.-H., Li, J., \& Liu, Y. 2007, Chin. J. Astron. and Astrophys., 7, 629 